\title{
Fourier Transforms as a Tool for Analysis of Hadron-Hadron Collisions
}

\author{
James Monk* \\ University College London \\ E-mail: jmonk@hep.ucl.ac.uk \\ Mario Campanelli \\ University College London \\ E-mail: mario.campanelli@hep.ucl.ac.uk
}

\begin{abstract}
Hadronic final states in hadron-hadron collisions are often studied by clustering final state hadrons into jets, each jet approximately corresponding to a hard parton. The typical jet size in a high energy hadron collision is between 0.4 and 1.0 in eta-phi. On the other hand, there may be structures of interest in an event that are of a different scale to the jet size. For example, to a first approximation the underlying event produces a uniform emission of radiation spanning the entire detector, colour connection effects between hard partons may fill the region between a jet and the proton remnant and hadronisation effects may extend beyond the jets. We consider the possibility of performing a Fourier decomposition on individual events in order to produce a power spectrum of the transverse energy radiated at different angular scales. We attempt to identify correlations in the emission of radiation over distances ranging from the full detector size to approximately 0.2 in eta-phi.
\end{abstract}

13th International Workshop on Advanced Computing and Analysis Techniques in Physics Research, ACAT2010

February 22-27, 2010

Jaipur, India

\footnotetext{
*Speaker.
} 


\section{Introduction}

QCD gives rise to many different features in collision events. Apart from hard scattering there is also radiation arising from initial and final state radiation, colour flow between the hard partons and a uniform contribution from underlying event. Identifying these features requires identifying radiation structures of different size in an event. The problem of separating objects of different size is not unique to high energy collider physics; in the field of cosmology, for example, a key observation is the angular size of correlations in the temperature and polarisation of the cosmic microwave background (CMB). This is studied by decomposing the image of the sky into a set of spherical harmonics [1]. Fourier transforms are also used in many other areas for signal processing.

One feature of QCD that can be hard to identify in the presence of the soft contribution from underlying event or multiple independent overlaid collisions is colour singlet exchange. In the normal production of jets in QCD there is colour flow between the jets, and the detector region between them is therefore populated with radiation. In colour singlet exchange there is a region of pseudo-rapidity, $\eta$, between the jets that is largely empty of radiation. This region is known as a rapidity gap and is also present in the exchange of other colour singlets, such as in vector boson fusion. Identifying the rapidity gap can be a powerful way to identify such events. However, underlying event and pile-up can emit radiation into the gap, which then becomes hard to observe. Since the contributions from underlying event and pile-up are uniformly distributed throughout the detector, but the gap (or conversely the colour connection effects) is more localised, one may hope that a Fourier analysis will be able to identify the presence of absence of a gap structure without being affected by other aspects of the event.

\section{Monte Carlo Sample, Event Selection and Fourier Transform}

Herwig [2] was used to generate four samples of Monte Carlo events: standard QCD di-jets and di-jets produced through colour singlet exchange, both with and without the underlying event model turned on [3]. Each event sample contained 15 million events. Herwig was modified for colour singlet exchange according to [4]. The Rivet [5] analysis package was used, which provides an interface between the generator event record and various analysis tools, including jet finding.

The KT jet algorithm [6] was run with an R parameter of 0.7 and events were selected that contained at least two jets with a jet transverse energy, $E_{T}$, above $30 \mathrm{GeV}$. The two leading jets were required to be separated by a pseudo-rapidity interval of at least $\Delta \eta>4$, both jets lying within $|\eta|<5$. The KT algorithm was run a second time with an R parameter of 0.1 in order to provide an input for the Fourier transform that ensures there can be no soft or collinear divergences. An $E_{T}$ cut of $1 \mathrm{GeV}$ was applied to these smaller jets.

The difference between colour singlet exchange and standard QCD di-jet production (henceforth QCD di-jets) can be seen in figure 1. In figure 1 the hardest jet is defined to be at $\phi=0$ and the $\phi$ position, weighted by $E_{T}$, of those KT 0.1 jets that lie between the two leading jets and nearer the hardest of the two leading jets is shown. The three curves show the effect of turning on first the underlying event model, which leads to a uniform increase in the $E_{T}$ deposited at all $\phi$, and the effect of turning on the colour connection between the two leading jets, which enhances the growth of $E_{T}$ in the $\phi$ region opposite the leading jet. This rise in radiation opposite the leading jet in $\phi$ is 


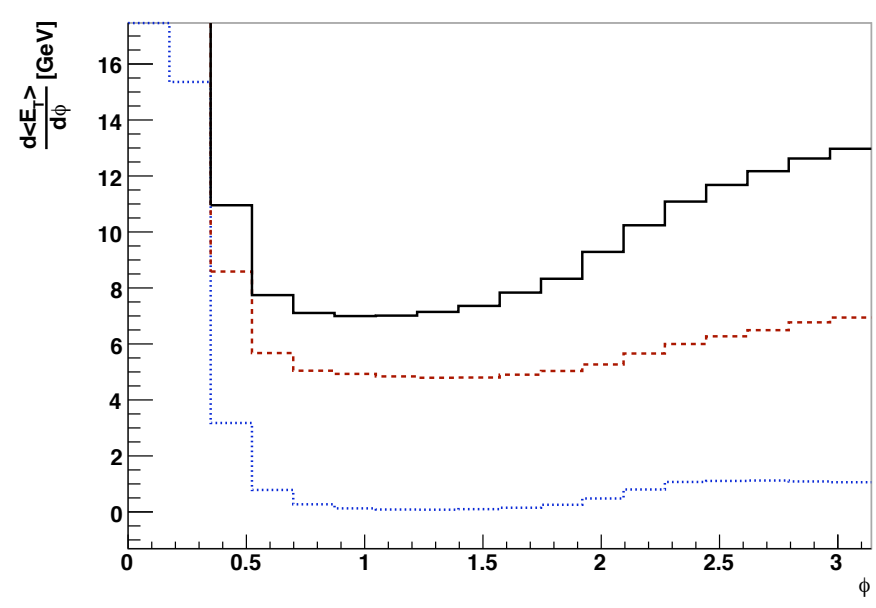

Figure 1: The $E_{T}$ flow in $\phi$ in the interjet region that is nearest the leading jet in $\eta$ (the leading jet is at $\phi=0$ ). The (blue) dotted line is colour singlet exchange and shows the smallest amount of radiation opposite the leading jet. The (red) dashed line shows colour singlet exchange with a uniform emission of radiation from underlying event. The (black) solid line is colour connected $\mathrm{d}$-jets with underlying event turned on, and shows an increase in the amount of radiation opposite the leading jet.

caused by showering outside of the next-to-leading jet, which is enhanced by the colour connection between the leading jets. This enhancement is of a different angular size to both the underlying event contribution and the hard jets.

The cylindrical coordinates of a hadron collision detector, together with the additive nature of rapidity under longitudinal boosts means that cylindrical harmonics are more appropriate than the spherical harmonics used in analyses of the CMB. The simplest Fourier transform that can be performed is the one-dimensional transform of the $E_{T}$ distribution in the $\phi$ coordinate, and it is this transformation that was performed in this study.

If one is satisfied to study only the magnitudes and not the phases of the Fourier coefficients then the location of $\phi=0$ event-by-event does not matter. However, different events have different orientations in $\phi$, and in order to study the phases of the coefficients the events must be lined up along a common axis. The position of $\phi=0$ is therefore defined to be the centroid of the hardest jet and the direction of increasing $\phi$ is defined such that the second hardest jet lies between $\phi=0$ and $\phi=\pi$. The coordinate system is divided up into a grid of $N=32$ bins and in each bin the sum of the $E_{T}$ s of the KT 0.1 jets is calculated. The KT 0.1 jets are restricted to lie with $|\eta|<5$. The GNU Scientific Library (GSL) [7] is used to provide a fast Fourier transform of the grid of 32 bins, which results in 16 independent complex Fourier coefficients.

\section{Results of the Fourier Transformation}

The sample of events produced through colour singlet exchange without a contribution from underlying event is the most purely di-jet like sample used; the other samples will exhibit more radiation outside of the leading pair of jets. Figure 2 shows the mean separate real and imaginary components of the Fourier coefficients from these most di-jet-like events. There is a clear alternation between the even coefficients, which are large (or positive, in the case of the imaginary part) and the odd coefficients, which are smaller than the even coefficients. Note also that the real part is much larger than the imaginary part. The reason for this is that whatever contribution a coefficient with odd coefficient number, $n$, makes at $\phi=0$, there will be an equally sized negative contribution 

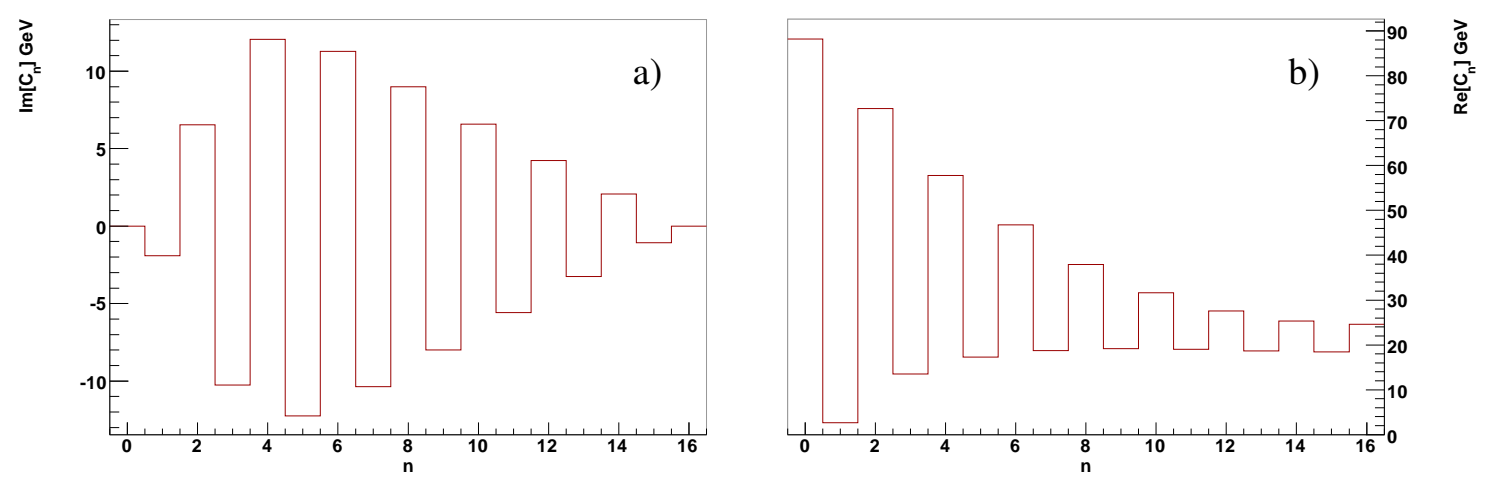

Figure 2: The imaginary (a) and real (b) parts of the Fourier coefficients averaged over 15 million di-jet events produced through the exchange of a colour singlet with no contribution from underlying event.

at $\phi=\pi$. The reverse is true of the even coefficients, which produce equal contributions at positions separated by an angle of $\pi$. Thus, for back-to-back di-jets the odd coefficients are suppressed relative to the even coefficients. The suppression becomes smaller for larger coefficient numbers, which correspond to smaller features. The fact that the odd coefficients are non-zero is because the next-to-leading jet has a lower $E_{T}$ than the leading jet, and the odd coefficients both soften and broaden the jet near $\phi=\pi$. The imaginary part is smaller than the real part because the events have been aligned so that the leading jet lies at $\phi=0$; the imaginary part produces no activity at $\phi=0$.

In figure 3 the average over 15 million events of the magnitudes of the Fourier coefficients are compared for colour singlet exchange events without underlying event turned on and QCD di-jets with underlying event. The QCD sample contains more inter-jet activity, which is apparent in figure 3 as a smaller suppression of the odd coefficients relative to the even coefficients.

The aim of the Fourier decomposition is to find new observables for distinguishing colour singlet exchange from QCD di-jets even in the presence of a contribution from underlying event. It has been demonstrated that the odd coefficients are more sensitive to inter-jet radiation than the even coefficients. The underlying event contribution, which is uniformly distributed, can be expected to affect mainly the smallest coefficients. Figure 4 shows the distribution of the $n=3$ coefficient for colour singlet events with and without the underlying event model switched on compared to the same coefficient from QCD di-jets in the presence of an underlying event contribution. There is little difference between the distributions from colour singlet exchange with and without underlying event, however the QCD di-jets distribution is distinct from the colour singlet distribution. The QCD di-jets tend to have a larger value of the $3^{\text {rd }}$ coefficient than the colour singlet exchange events.

\section{Summary}

We have shown that the colour connection properties of different types of event produce different distributions of the azimuthal Fourier transform coefficients. The only contribution to the odd Fourier coefficients comes from deposits of radiation that are not back-to-back di-jet like. The 

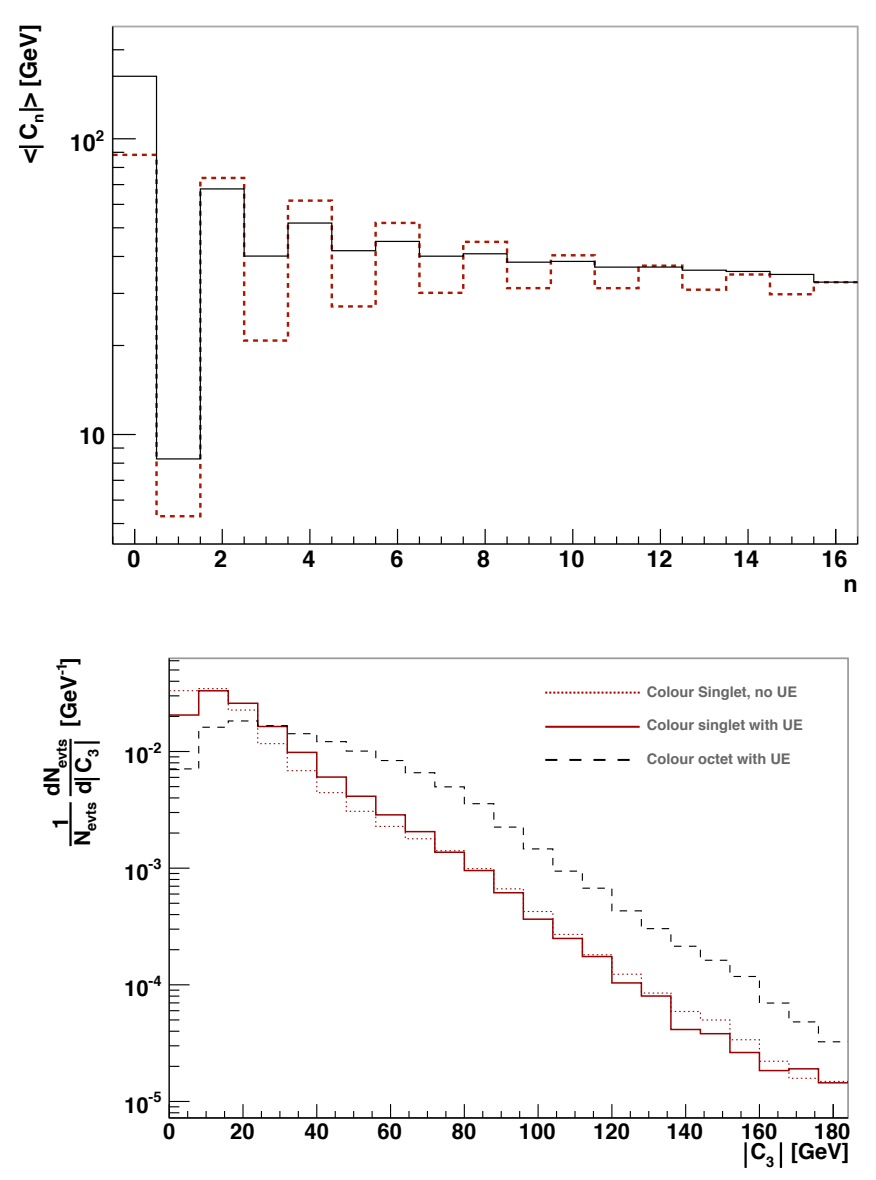

Figure 3: The magnitudes, averaged over 15 million events, of the Fourier coefficients for QCD dijets with underlying event turned on (black solid) compared with colour singlet di-jets without a contribution from underlying event (dashed red). The QCD di-jets, which contain more inter-jet radiation, show larger values for the odd coefficients

Figure 4: The distribution of the magnitude of the third Fourier coefficient for colour singlet events with (red solid) and without (red dotted) a contribution from underlying event and QCD events with an underlying event contribution (black dashed). The presence or absence of underlying event makes little difference to the colour singlet distributions, yet there is a difference between the QCD and colour singlet curves.

underlying event also contributes to inter-jet radiation, however, due to its uniform nature it contributes more to the $0^{\text {th }}$ Fourier coefficient than any other. Thus an odd coefficient such as the $3^{\text {rd }}$ coefficient shows sensitivity to colour connection effects in the inter-jet region without being affected by the uniform distribution of activity from the underlying event.

\section{References}

[1] M. R. Nolta et al. Five-Year Wilkinson Microwave Anisotropy Probe (WMAP) Observations: Angular Power Spectra. Astrophys. J. Suppl., 180:296-305, 2009.

[2] G. Corcella et al. HERWIG 6.5 release note. hep-ph/0210213, 2002.

[3] J. M. Butterworth, J. R. Forshaw, and M. H. Seymour. Multiparton interactions in photoproduction at HERA. Z. Phys., C72:637-646, 1996.

[4] B. Cox, J. R. Forshaw, and L. Lonnblad. Hard color singlet exchange at the Tevatron. JHEP, 10:023, 1999.

[5] B. M. Waugh, H. Jung, A. Buckley, L. Lonnblad, J. M. Butterworth and E. Nurse, arXiv:hep-ph/0605034.

[6] M. Cacciari and G. P. Salam. Dispelling the $N^{3}$ myth for the $k_{t}$ jet-finder. Phys. Lett., B641:57-61, 2006.

[7] http://www.gnu.org/software/gsl/manual/html_node/. 